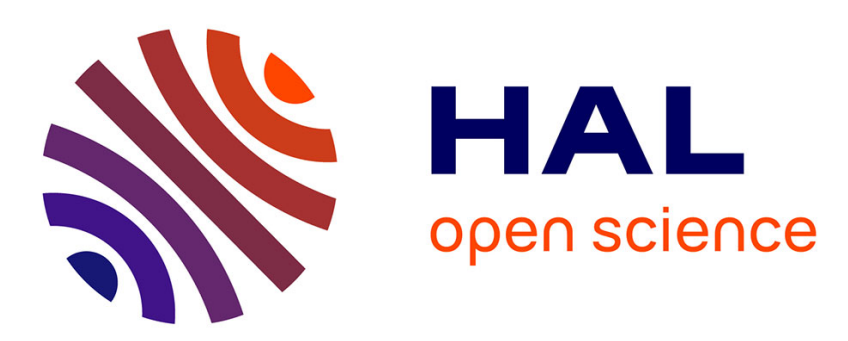

\title{
Analysis and Comparison of Functional Dependencies of Multiscale Textural Features on Monospectral Infrared Images
}

\author{
Jacopo Grazzini, Hussein Yahia, Isabelle Herlin, Antonio Turiel
}

\section{To cite this version:}

Jacopo Grazzini, Hussein Yahia, Isabelle Herlin, Antonio Turiel. Analysis and Comparison of Functional Dependencies of Multiscale Textural Features on Monospectral Infrared Images. IGARSS IEEE International Geoscience and Remote Sensing Symposium, Jul 2003, Toulouse, France. pp.20452047, 10.1109/IGARSS.2003.1294334 . hal-01302802

\section{HAL Id: hal-01302802 \\ https://inria.hal.science/hal-01302802}

Submitted on 15 Apr 2016

HAL is a multi-disciplinary open access archive for the deposit and dissemination of scientific research documents, whether they are published or not. The documents may come from teaching and research institutions in France or abroad, or from public or private research centers.
L'archive ouverte pluridisciplinaire HAL, est destinée au dépôt et à la diffusion de documents scientifiques de niveau recherche, publiés ou non, émanant des établissements d'enseignement et de recherche français ou étrangers, des laboratoires publics ou privés. 


\section{Analysis and Comparison of Functional Dependencies of Multiscale Textural Features on Monospectral Infrared Images}

\author{
Jacopo Grazzini, Hussein Yahia and Isabelle Herlin \\ Air Project \\ INRIA Rocquencourt \\ Domaine de Voluceau BP105, 78153 Le Chesnay Cedex, France \\ \{jacopo.grazzini,hussein.yahia,isabelle.herlin\}@inria.fr
}

\author{
Antonio Turiel \\ Departament de Física Fonamental \\ Universitat de Barcelona \\ Diagonal, 647. 08028 Barcelona, Spain \\ turiel@ffn.ub.es
}

\begin{abstract}
In this paper, we deal with the problem of extracting meaningful textural features leading to good segmentations on satellite images of natural environments. Standard texture features using graylevel co-occurrence matrices have been widely applied on remote sensed images but they impose limitations (due to finite window sizes) as poor spatial localization. We have generalized the definition of texture features using a multiscale framework, in order to take advantage of multiscale properties of natural images. The new definition improves spatial localization and the relevance of the parameters. We then investigate the dependencies among different features for classification purposes. An unsupervised scheme of classification was performed on different satellite infrared images. We see that natural, chaotic images should be treated with a different methodology.
\end{abstract}

\section{INTRODUCTION}

Texture segmentation is one of the central problems in image processing and has given rise to an abundant scientific literature [11]. Techniques which use textural (combined with spectral) information are among the methods which have given the best results in satellite imagery [12].The main problem in the segmentation of natural environment images is the large variability of texture characteristics over them.

Segmentation of images is usually performed in two stages [9]. In a first stage, features characterizing the texture are calculated. In a second stage, those features are used to determine uniform regions over the image. The main purpose of texture feature extraction is to find relations among pixels belonging to a similar texture. As satellite images display fine-grained textures, a statistical approach is often adopted for remotely sensed data: statistical measures of the spatial distribution of graylevels are computed. The most common method consists in computing local co-occurrence matrices representing joint probabilities of graylevel pairs [8] and from that to derive some statistical measures [13]. Classification schemes based on those methods provide good results in cloud classification [13] [7] or land cover segmentation [5], and show also good performance on benchmark images [11].

In this paper, we focus on the use of multiscale textural features for the segmentation of infrared images of natural environments. We show the limitation of a classification scheme on meteorological images, as the features appear to be mutually functionally dependent. In the next section, we introduce the concept of multiscale textural features which generalize the classical definition. In section III, we present measures of functional correlation between features computed on Landsat, Spot and MeteoSat infrared images. We perform then a classical K-Means classification on those data in section IV and we interpret the different results in section V.

\section{Multiscale textural features}

Information in a natural image is not contained at only one scale: multiple objects of different real and apparent sizes appear intervowed in a complicated mesh. It is thus necessary to relate somehow information from the different scales of resolution. Approaches based on co-occurrence matrices, generally obtained over fixed size windows, need to be extended in order to acquire textural features at several scales [10].

We propose to generalize co-occurrence in a multiscale framework by introducing a non-uniform, scale-invariant weighting function in the computation of spatial distribution of grey-levels variations. The standard way for the evaluation of gray-level distribution consists in defining small (overlapping or not) windows of predefined size around each pixel, then computing the relative frequency of the observed pairwise graylevels and finally calculating a representative feature (GLCM approach [13]). In our approach, instead of defining a small window around the pixel $x$, we consider a rather large window $W(x)$ but each pair of graylevels is assigned a weight so that pairs of pixels further and further away will contribute less and less. In such a way, a good localization is obtained, even for large windows. We define the multiscale joint probability $p_{i j}^{W}(x)$ of a graylevel pair $(i, j)$ by: $p_{i j}^{W}(x) \propto$ $\sum_{y, y^{\prime} \in W(x) \mid I(y)=i, I\left(y^{\prime}\right)=j}\left|x-\left(y+y^{\prime}\right) / 2\right|^{-\alpha}$. We choose the exponent $\alpha=2$. Due to the scale invariant character of this weight function, the result does not in principle depend on the size of the window [6], although we limit the calculations to a $21 \times 21$ window to avoid divergences and to fasten calculations. For that reason, the computation of the features [13] does not depend either on any fixed scale: it is scale invariant. This 
method [9]. The results of the segmentations on land-cover images can be compared with those based on all spectral channels (Fig. 3); textural features allow to characterize some well textured regions (fields and water) but have difficulty to extract small textured areas (cities). For MeteoSat images, the segmentations are not so good, and they are not improved when new features are included in the classification procedure (Fig. 4).
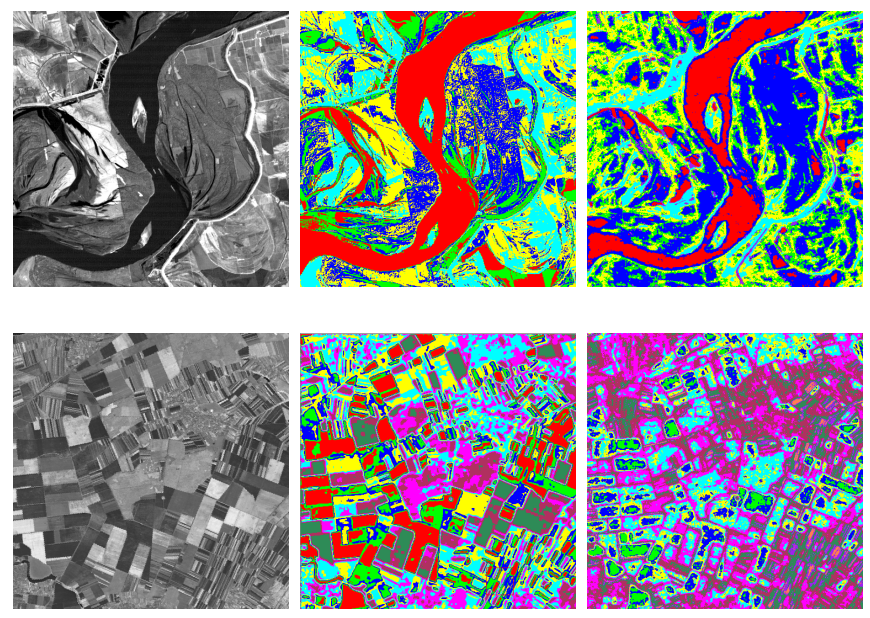

Fig. 3. Left: Landsat (top) and Spot (bottom) NIR land-cover images. Middle: K-Means classification with spectral features. Rigth: K-Means classification with textural features.

\section{DisCUSSION AND CONCLUSION}

We have investigated the mutual dependencies of multiscale features when computed on two types of infrared images: land cover images (from Spot and LandSat satellites) and higher atmosphere temperature images (from MeteoSat). We see that for land-cover images the different textural features are dependent on the underlying region, what allows to classify those regions by applying standard algorithms (as K-means) in the feature space. This dependence of the features on the spatial region is evidenced by the weak functional dependence among features (measured by the mutual information and correlation ratio) and by the multi-valued character of the conditional distributions (as different textures are represented by different clusters in feature space).

On the contrary, segmentation methods based on texture extraction do not work when applied to MeteoSat IR data,
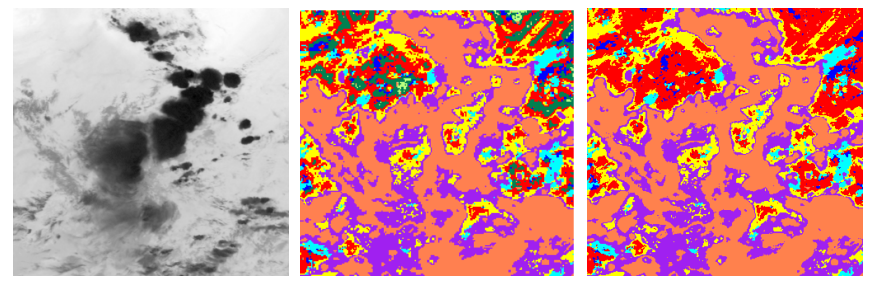

Fig. 4. Left: MeteoSat IR image. Middle: K-Means classification with textural features. Right: segmentation obtained with entropy feature only. a fact already pointed out by $\mathrm{Gu}$ [7] and Ebert [4] in the classification of clouds. One of the reasons of this failure lies in the fact that those methods assume regularity conditions that are not satisfied by MeteoSat images (those images are related to thermodynamical properties of a turbulent, chaotic flow). A more detailed analysis of the features shows a remarkable degree of mutual dependency among features, together with narrow, uni-valued conditional distributions of pairs of features. This dependency means that all the features are sensitive to the same property of images and multiple feature classification does not provide new meaningful features. Henceforth, the segmentation has to be carried out by means which take into account the properties of the flow, as for instance performing multiscale singularity analysis [6].

To conclude, the results shown so far means that, unlike what is discussed in [1], there is not an image-independent methodology for feature selection, and in particular classification techniques on multi-feature spaces do not work efficiently for every kind of data disregarding inherent structure of images. Methodologies which are related to the properties of the object of study, specially in the case of natural, chaotic images, should be considered.

\section{ACKNOWLEDGMENT}

We thank the Laboratoire de Météorologie Dynamique (École Polytechnique, France) for the data. We are also grateful to B. Guldner for his helpful comments. J. Grazzini is funded by a grant from the Regional Council of Île-de-France (agreement no. E.1358). A. Turiel is funded by a contract RED2002 from Generalitat de Catalunya.

\section{REFERENCES}

[1] A. Baraldi and F. Parmiggiani, An investigation of the textural characteristic associated with gray level cooccurrence matrix statistical parameters, IEEE Trans. GRS, 33(2):293-304, 1995.

[2] B. Baum et al., Automated cloud classification of global AVHRR data using fuzzy logic approach, J. Appl. Meteor., 36(11):1519-1533, 1997.

[3] T. M. Cover and J. .A. Thomas, Elements of Information Theory, John Wiley, New York, 1991.

[4] E. Ebert, Analysis of polar clouds from satellite imagery using pattern recognition and a statistical cloud analysis scheme, J. Appl. Meteor., 28:382-399, 1989.

[5] P. Gong, J. D. Marceau and P. J. Horwarth, A comparison of spatial feature extraction algorithms for land-use classification with SPOT HRV data, Remote Sens. Envir., 40:137-151, 1992.

[6] J. Grazzini, A. Turiel and H. Yahia, Entropy Estimation and Multiscale Processing in Meteorological Satellite Images, Proc. of ICPR, 3:764-768, 2002.

[7] Z. Gu et al., Textural and spectral features as an aid to cloud classification, Int. J. of Remote Sens., 12(5):953-968, 1991.

[8] R. Haralick, K. Shanmugam and I. Dinstein, Textural features for image classification, IEEE Trans. SMC, 3(6):610-621, 1973.

[9] A. Jain and R. Dubes, Algorithms for clustering data, Prentice Hall, Englewood Cliffs, New Jersey, 1988.

[10] V. Metzler et al., Texture classification of graylevel images by multiscale cross-cooccurrence matrices, Proc. of ICPR, 4:549-552, 2000.

[11] T. Randen and J. Husoy, Filtering for texture classification: a comparative study, IEEE Trans. PAMI, 21(4):291-310, 1999.

[12] M. Shokr, Evaluation of second-order texture parameters for sea ice classification in radar images, J. Geophys. Res., 96(6):10625-10640, 1991.

[13] R. Welch, S. Sengupta and D. Chen, Cloud field classification based upon high spatial resolution textural features. Part I: Gray level cooccurrence matrix approach, J. Geophys. Res., 93(10):12663-12681, 1988. 